\title{
Uterine Artery Embolization Improves Quality of Life in Patients with Pure Adenomyosis: A Single-Center Experience
}

\begin{abstract}
Aim: The aim of the study was to assess clinical outcomes following uterine artery embolization (UAE) in the treatment of dysmenorrhea and menorrhagia related to pure adenomyosis. Materials and Methods: This was a retrospective analysis of 14 patients with dysmenorrhea and menorrhagia related to pure adenomyosis treated with UAE using polyvinyl alcohol (PVA) between January 2017 and September 2019. The baseline and 3-month and 12-month postintervention outcomes were assessed using Uterine Fibroid Symptom-Health-Related Quality of Life Questionnaire (UFS-QOL), Symptom Severity Score (SSS), and magnetic resonance imaging findings. The median age of the patients was 47 years (28-55). The main clinical presentation was dysmenorrhea and menorrhagia, with a median duration of symptom of 24 (12-84) months. All patients received combined estrogen-progestin and nonsteroidal anti-inflammatory drugs prior to UAE without clinical improvement. Results: According to UFS-QOL and SSS results, the score for distress level was statistically significantly reduced from baseline 4.6 (3.6-5) to 2.25 (1-3.37) $(P=0.018)$ after 3 months, and remained stable 12 months later. The score of symptom level was statistically significantly reduced from $4(3.6-5)$ at baseline to $1.6(1-3.58)(P=0.018)$ after 3 months with maintained clinical satisfaction after 12-month follow-up. The junctional zone was statistically significantly reduced from baseline with a median of $33.5 \mathrm{~mm}(19-79 \mathrm{~mm})$ to $25 \mathrm{~mm}$ $(8-77 \mathrm{~mm})$ after 3 and 12 months $(P=0.046)$. The incidence of post-UAE permanent amenorrhea is $71 \%$ with a mean age of 49 years. No patient underwent hysterectomy during the follow-up period. Conclusion: UAE using PVA can improve the quality of life in patients with menorrhagia and dysmenorrhea related to adenomyosis. However, larger prospective studies are needed to establish the long-term outcomes and risk of amenorrhea.
\end{abstract}

Keywords: Adenomyosis, dysmenorrhea and uterine fibroid symptom and health-related quality of life, menorrhagia, uterine artery embolization

\section{Introduction}

Adenomyosis is defined by Bird in 1972 as "the benign invasion of endometrium into the myometrium, producing a diffusely enlarged uterus which microscopically exhibits ectopic nonneoplastic, endometrial glands and stroma surrounded by the hypertrophic and hyperplastic myometrium."[1] Adenomyosis can be asymptomatic. However, patients frequently present with symptoms of menorrhagia, dysmenorrhea, and other possible symptoms, such as dyspareunia, chronic pelvic pain, and irritation of the urinary bladder. The condition is typically found in women between the ages of 35 and 50 , but also affects younger women. Treatment options consist of hormonal therapy, endometrial ablation, and uterine

\footnotetext{
This is an open access journal, and articles are distributed under the terms of the Creative Commons Attribution-NonCommercial-ShareAlike 4.0 License, which allows others to remix, tweak, and build upon the work non-commercially, as long as appropriate credit is given and the new creations are licensed under the identical terms.
}

For reprints contact: WKHLRPMedknow_reprints@wolterskluwer.com artery embolization (UAE). However, when these therapies fail, hysterectomy may be considered as a definitive treatment. ${ }^{[2,3]}$ UAE was first described in 1995 for the treatment of uterine fibroids (UFs). ${ }^{[4]}$ It has been established as a valuable treatment option for patients with symptomatic UFs. ${ }^{[5,6]}$ Since then, UAE has been tried as a possible treatment option for adenomyosis and seems to have a favorable outcome in multiple case series. ${ }^{[7,8]}$ The purpose of this study is to assess the clinical outcomes of UAE in the treatment of symptomatic adenomyosis.

The symptoms of adenomyosis are quite similar to that of UF, and their negative impact on health-related quality of life (HRQoL) makes women seek therapy for adenomyosis. Therefore, patient-reported outcome measures are considered appropriate tools to measure

\footnotetext{
How to cite this article: Alrashidi I, Alharthy $\mathrm{H}$, Alahmari F, Alammari S, Alobaidi H, Alruhaimi A, et al. Uterine artery embolization improves quality of life in patients with pure adenomyosis: A single-center experience. Arab J Intervent Radiol 2020;4:117-20.
}

\author{
Ibrahim Alrashidi', \\ Hayat Alharthy², \\ Faisal Alahmari ${ }^{1}$, \\ Sultan Alammari ${ }^{1}$, \\ Hatim Alobaidi ${ }^{1}$, \\ Abdulwaeed \\ Alruhaimi ${ }^{1}$, \\ Abdulaziz \\ Almat'hami', \\ Abdulrahman \\ Alkhalifah ${ }^{1}$, \\ Fares Garad ${ }^{1}$
}

Departments of ${ }^{\prime}$ Radiodiagnostics and Medical Imaging and ${ }^{2}$ Obstetric and Gynecology, Prince Sultan Military Medical City, Riyadh, Saudi Arabia

Received: $12-11-2019$ Revised: 03-04-2020 Accepted: 10-04-2020 Online Published: 17-08-2020

Address for correspondence: Dr. Ibrahim Alrashidi, Prince Sultan Military Medical City, Riyadh, Saudi Arabia. E-mail:dr.Ialrashidi@gmail. com

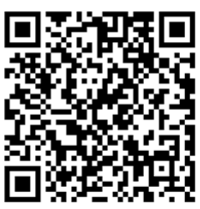


the impact and outcome of interventions. ${ }^{[9]}$ The Uterine Fibroid Symptom and Health-Related Quality of Life Questionnaire (UFS-QOL) is widely used to evaluate patient-reported UF symptoms and their impact on HRQOL, which is the only disease-specific instrument developed and validated in a population of women with UFs, which is believed to be used in adenomyosis study with similar symptoms. ${ }^{[9]}$ This procedure was developed based on qualitative input from patients with UFs; the original validation demonstrated its ability to discriminate between women with and without UFs and also between varying patient-reported disease severity as well. ${ }^{[10]}$ Moreover, the UFS-QOL has been shown to be highly responsive and adaptive to change following treatment. ${ }^{[9]}$

\section{Materials and Methods}

The institutional review board has approved this retrospective study, and informed consent was waived. Patients who underwent UAE for adenomyosis between January 2017 and September 2019 were included. This single-study experience aims to evaluate the role of UAE as a treatment option to relieve clinical symptoms associated with adenomyosis in patients who failed combined estrogen-progestin and nonsteroidal anti-inflammatory drug treatment, and in women who sought to preserve the uterus or in nonsurgical candidate treatment.

A total of 14 women were enrolled with adenomyosis; two of them were single, nulliparous patients. The median age of the women was 47 years (28-55 years). All participants had menorrhagia and dysmenorrhea with a median duration of 24 months (12-84). All women had persistent symptoms after receiving combined estrogen-progestin and nonsteroidal anti-inflammatory drug treatment for an average of 12 months.

Patients were diagnosed with adenomyosis with a median junctional zone thickness of $33.5 \mathrm{~mm}$ (19-79), based on magnetic resonance imaging (MRI) with a follow-up period of 3 months or longer. The mean follow-up period following UAE was 6.5 months (range 3-12 months). Short-term post-UAE follow-up consisted of an outpatient visit and pelvic MRI at 3 and 12 months. The diagnosis was established clinically and confirmed with MRI.

Pelvic MRI consisted of T2- and T1-weighted contrast-enhanced images and was performed before embolization and at 3-12 months post-UAE. MRI findings were blindly reviewed by two radiologists, and the following standardized criteria were used for the diagnosis of adenomyosis: low myometrial signal intensity on T2-weighted images and diffuse or focal thickening of the junction zone exceeding $12 \mathrm{~mm}$ with or without high signal intensity foci corresponding to myometrial cysts. ${ }^{[1,2]}$ At 3- and 12-month follow-up, the decrease in junction zone was measured on the MRI post treatment. MRI was used to assess the uterine volume at baseline and at 3-12 months
post-UAE, using the formula of an ellipsoid which uses three mutually perpendicular axes that intersect at the center.

UAE was performed after selective catheterization of both uterine arteries through right common femoral approach using $355-500-\mu$ ( $n=11$ patients $) / 500-700-\mu$ ( $n=3$ patients $)$ polyvinyl alcohol (PVA) (Contour, Boston Scientific, Natick, USA) particles until complete stasis. During and after UAE, intravenous narcotics and antiemetic drugs were administered for adequate pain control and to reduce symptoms, such as nausea and vomiting. All patients received intravenous $1 \mathrm{~g}$ cefazoline $1 \mathrm{~h}$ before UAE and ciprofloxacin $500 \mathrm{mg}$ orally, twice daily for 10 days after $\mathrm{UAE}$ as prophylaxis.

On the day of admission, immediately prior to UAE, baseline clinical status was assessed using the UFS-QOL, which consists of a Symptom Severity Score (SSS) and HRQOL score. ${ }^{[9-11]}$ The UFS-QOL questionnaire consists of an 8-item symptom severity scale containing questions addressing topics, such as menstrual bleeding characteristics, pelvic pain, urinary discomfort, and fatigue. It also contains $29 \mathrm{HRQoL}$ items comprising the following six domains: concern, activities, energy/mood, control, self-consciousness, and sexual function. ${ }^{[3,4]}$ The same questionnaire was used at post-UAE period of 3 and 12 months. Women with a SSS $<20$ in combination with an overall health-related QOL score $>80$ were considered asymptomatic.

Data results were analyzed by using descriptive and inferential statistics through IBM SPSS version 20 (IBM Corp., Armonk, NY, USA). Descriptive statistics, including median (minimum-maximum), were calculated for continuous variables such as age, uterus volume, junctional zone, and duration of symptoms. Categorical variables such as clinical presentation, hormonal therapy, and MRI imaging findings were presented as frequencies and percentages, $n(\%)$. Uterine volume and junctional zone reduction 3 and 12 months following UAE were compared to baseline. A Wilcoxon signed-rank test was applied to compare variables across pre- and post-UAE after a follow-up period of 3 and 12 months. $P<0.05$ was considered statistically significant.

\section{Results}

UAE procedures are $100 \%$ technically successful; no immediate complication has been recorded.

The baseline score for distress level was high prior to UAE procedure, reaching 4.6 (3.6-5) according to UFS-QOL, which was significantly reduced to 2.25 (1-3.37) $(P=0.018) 3$ months after UAE, and remained stable (2.19 [1-3.39]) after 12-month follow-up [Figure 1]. The score of symptoms level statistically significantly reduced $(P=0.018)$ from baseline $4(3.6-5)$ to 1.6 (1-3.58) 3 months following UAE and remained stable (1.7 [1-3.62]) after 12-month follow-up [Figure 2]. 
The uterine volume showed no statistically significant change between baseline with a median of $462 \mathrm{ml}$ and 3 months following UAE with a median of $205.4 \mathrm{ml}$ $(P=0.176)$, and these results are skewed by the single case of very large volume. The junctional zone $(\mathrm{mm})$ showed statistically significant reduction from baseline $(19-79 \mathrm{~mm})$ to $(8-77 \mathrm{~mm})(\mathrm{P}=0.046)$ after 3 and 12 months as shown in Figure 3 and Table 1.

Permanent amenorrhea, defined as the absence of menstrual periods for at least 12 months post-UAE, occurred in $10 / 14$ patients $(71 \%)$, with a mean age of 49 years. No patient underwent hysterectomy during the follow-up period.

\section{Discussion}

The most noticeable improvement of HRQOL in patients with adenomyosis occurred in the first 3 months post-UAE and remained stable at 12 months. This finding is concordant with a meta-analysis of 15 studies, which showed symptomatic improvement in $75.7 \%(387 / 511)$ at a median follow-up of 26.9 months. $^{[7]}$

PVA particles measuring 355-500 $\mu$ have been used in patients with symptomatic adenomyosis with promising clinical results. ${ }^{[12]}$ This study has demonstrated that PVA can be used with similar good clinical response in patients with symptomatic adenomyosis. PVA was selected for three reasons: availability, operator familiarity, and cheaper price compared to other embolic agents, and the small particle size was chosen for deeper penetration into the small arterioles to achieve the required tissue penetration. In this small sample study, 10 of 14 women experienced primary amenorrhea 3-6 months following UAE, with a mean age of 49 years. This is higher than what was previously reported in the meta-analysis by Popovic et al. $(20.9 \%)^{[7]}$ and the meta-analysis by de Burijn et al., which showed persistent amenorrhea in 28 of 445 patients $(6.3 \%)$ in 13 studies. However, patients who suffered amenorrhea in the prior studies were older than 45 years $^{[7]}$ and 40 years of age, ${ }^{[8]}$ and this may explain the higher rate of amenorrhea in the present study due to the older mean age. In this small sample study, the symptom and distress level associated with adenomyosis clinically subsided according to post-UAE UFS-QOL, which is concordant with Popovic's meta-analysis review. ${ }^{[7]}$

This study has a number of limitations including its retrospective nature, small number of patients, and the inherent section bias. Therefore, strong conclusions cannot be drawn about what type of adenomyosis or symptoms may respond best. Second, many patients experienced post-UAE amenorrhea that could be related to the older mean age.

The third limitation is the absence of long-term data, limiting the ability to measure the durability of UAE to other medical or surgical treatments. However, UAE

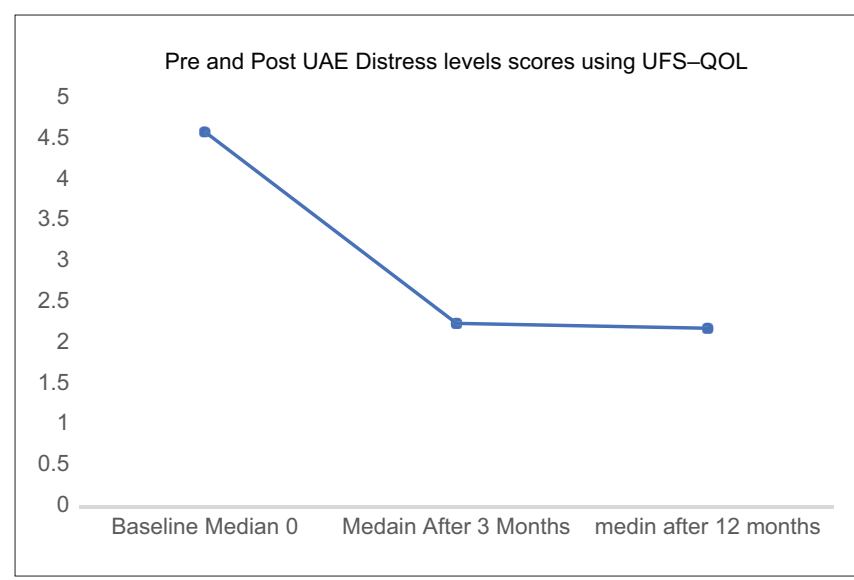

Figure 1: Distress level scores using Uterine Fibroid Symptom-Health-Related Quality of Life Questionnaire: Pre (baseline) and post (after 3 and 12 months) uterine artery embolization follow-up

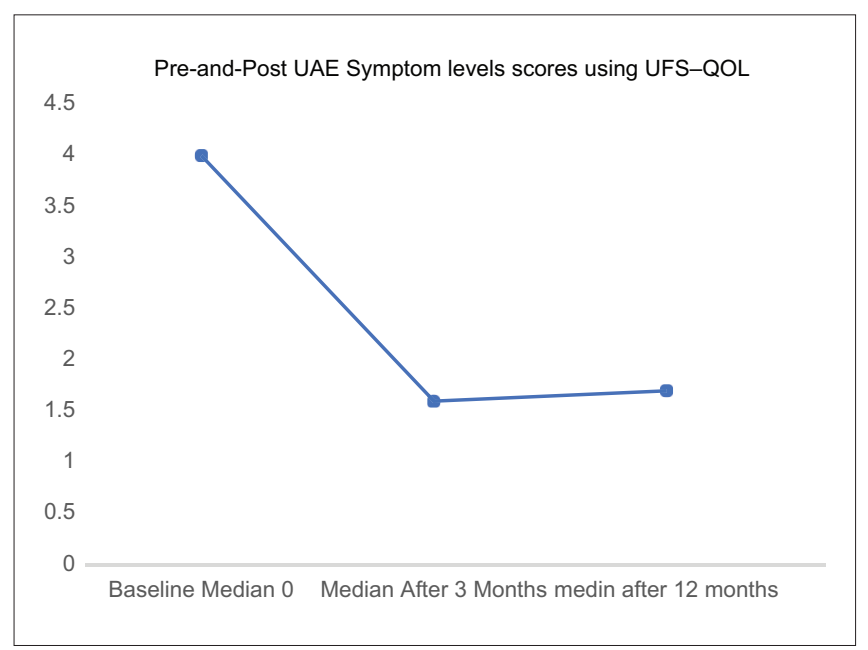

Figure 2: Symptom level scores using Uterine Fibroid Symptom and Health-Related Quality of Life Questionnaire pre (baseline) and post (after 3 and 12 months) uterine artery embolization follow-up

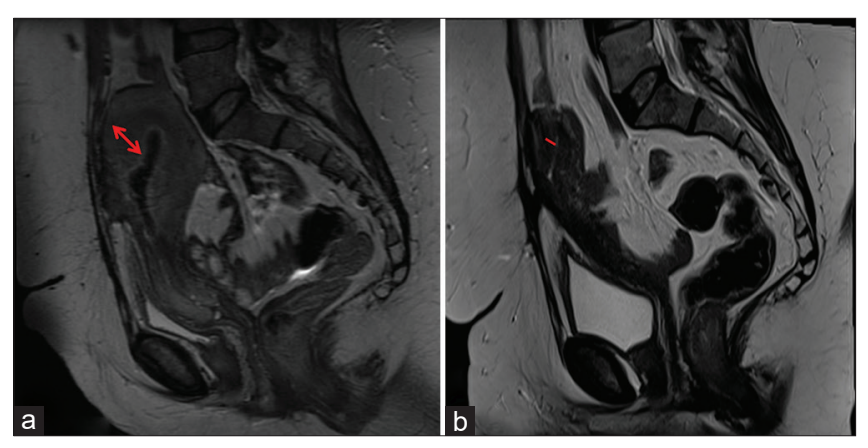

Figure 3: A 45-year-old patient with dysmenorrhea and menorrhagia related to adenomyosis sagittal pelvic magnetic resonance imaging T2. (a) Preuterine artery embolization junctional zone measures $19 \mathrm{~mm}$ (two-head arrows), (b) 3-month postuterine artery embolization junctional zone measures $8 \mathrm{~mm}$ (red line)

can improve the symptoms and distress associated with adenomyosis, which, in turn, can improve the patient's quality of life. Larger prospective studies are needed to establish the long-term outcomes and risk of amenorrhea. 
Table 1: Pre (baseline) and post (after 3- and 12-month follow-up) uterine volume and junctional zone

\begin{tabular}{lccc}
\hline Variable & \multicolumn{2}{c}{ Median (minimum-maximum) } & \multicolumn{1}{c}{$\boldsymbol{P}$-Value } \\
\cline { 2 - 4 } & Pre & Post 3-month follow-up & Post 12-month follow-up \\
\hline Whole uterus (ml) & $462(381-3816)$ & $205.4(114.4-2714.7)$ & $200.9(110.7-2689.5)$ \\
Junctional zone (mm) & $33.5(19-79)$ & $25(8-77)$ & $24(8-79)$ \\
\hline
\end{tabular}

${ }^{a}$ Wilcoxon signed-rank test

\section{Conclusion}

UAE using PVA can improve the quality of life in patients with menorrhagia and dysmenorrhea related to adenomyosis. However, larger prospective studies are needed to establish the long-term outcomes and risk of amenorrhea.

\section{Acknowledgments}

1. The UFS-QOL questionnaire was obtained from the SIR foundation. We would like to show our gratitude to the SIR foundation for sharing their pearls of wisdom with us during this research, although they may not agree with all of the interpretations/conclusions of this article

2. We thank Dr. Yahya Mahali, body imaging fellow, for assistance with the methodology

3. We would also like to show our gratitude to Rawabi M. Alsayer, Research Officer in Scientific Research Center at Prince Sultan Military Medical City, Riyadh, Kingdom of Saudi Arabia, for helping us in the statistical analysis during the course of this research.

Financial support and sponsorship

Nil.

\section{Conflicts of interest}

There are no conflicts of interest.

\section{References}

1. Bird CC, McElin TW, Manalo-Estrella P. The elusive adenomyosis of the uterus-revisited. Am J Obstet Gynecol 1972;112:583-93.

2. Li JJ, Chung JP, Wang S, Li TC, Duan H. The Investigation and management of adenomyosis in women who wish to improve or preserve fertility. Biomed Res Int 2018;2018:6832685.

3. Azziz R. Adenomyosis: Current perspectives. Obstet Gynecol Clin North Am 1989;16:221-35.

4. Ravina JH, Herbreteau D, Ciraru-Vigneron N, Bouret JM, Houdart E, Aymard A, et al. Arterial embolisation to treat uterine myomata. Lancet 1995;346:671-2.

5. de Bruijn AM, Ankum WM, Reekers JA, Birnie E, van der Kooij SM, Volkers NA, et al. Uterine artery embolization versus hysterectomy in the treatment of symptomatic uterine fibroids: 10-year outcomes from the randomized EMMY trial. Am J Obstet Gynecol 2016;215:745.e1-e12.

6. Goodwin SC, Spies JB, Worthington-Kirsch R, Peterson E, Pron G, Li S, et al. Uterine artery embolization for treatment of leiomyomata: Long-term outcomes from the FIBROID Registry. Obstet Gynecol 2008;111:22-33.

7. Popovic M, Puchner S, Berzaczy D, Lammer J, Bucek RA. Uterine artery embolization for the treatment of adenomyosis: A review. J Vasc Interv Radiol 2011;22:901-9.

8. de Bruijn AM, Smink M, Lohle PN, Huirne JA, Twisk JW, Wong $\mathrm{C}$, et al. Uterine artery embolization for the treatment of adenomyosis: A systematic review and meta-analysis. J Vasc Interv Radiol 2017;28:1629-42.e1.

9. Coyne KS, Margolis MK, Bradley LD, Guido R, Maxwell GL, Spies JB. Further validation of the uterine fibroid symptom and quality-of-life questionnaire. Value Health 2012;15:135-42.

10. Spies JB, Coyne K, Guaou Guaou N, Boyle D, Skyrnarz-Murphy K, Gonzalves SM. The UFS-QOL, a new disease-specific symptom and health-related quality of life questionnaire for leiomyomata. Obstet Gynecol 2002;99:290-300.

11. Harding G, Coyne KS, Thompson CL, Spies JB. The responsiveness of the Uterine Fibroid Symptom and Health-Related Quality of Life Questionnaire (UFS-QOL). Health Qual Life Outcomes 2008;6:99.

12. Kim MD, Kim S, Kim NK, Lee MH, Ahn EH, Kim HJ, et al. Long-term results of uterine artery embolization for symptomatic adenomyosis. AJR Am J Roentgenol 2007;188:176-81. 